\title{
Patrick Le Roux, Mémoires hispaniques. Essai sur la pratique de l'Histoire, Madrid: Casa de Velázquez, 2012, 212 pags.
}

Podemos afirmar que el análisis historiográfico no ha gozado de tanto predicamento en el ámbito de los estudios de la antiguiedad como en otras parcelas de la investigación histórica. Así pues, este libro del profesor Le Roux, ahora catedrático emérito en la Université de Paris 13, que constituye el cuarto volumen de la serie "Essais de la Casa de Velázquez", tiene un carácter un tanto excepcional a pesar de haber sido elaborada por un académico de un país tan prolífico en estudios de historiografía como es Francia.

El propio título de la monografía ya anuncia la naturaleza en cierto modo ecléctica y profundamente personal del estudio, cuyo índice recopila una serie de cuestiones que el autor juzga centrales, tanto para el conocimiento del mundo romano específicamente hispano, como para el conjunto de la realidad provincial. Sin embargo, esta obra no es sólo un mero recorrido a través de algunos de los principales problemas de la antigüedad hispanorromana, sino más bien un compendio de reflexiones sobre el trabajo de historiador nacidas de las experiencias en España del propio autor durante su dilatada carrera, fundamentalmente a partir del momento en el que se incorpora a la Casa de Velázquez en 1970. En definitiva, un repaso a la historiografía sobre Hispania y el mundo romano hecha en las cuatro últimas décadas. Especial atención le merecen al investigador francés las cuestiones de método y las metodologías, así como los distintos paradigmas y modelos interpretativos desde los que sucesivos investigadores han abordado la problemática tratada en esta obra, sin que tenga problemas en etiquetar algunos enfoques como historicistas, estructuralistas o marxistas. De esta manera, junto a las numerosas alusiones a autores clásicos como Casio Dión, Apiano o Estrabón y a los pertinentes estudiosos modernos de la antigüedad, el texto aparece también trufado de referencias a Claude Lévi-Strauss, Hanna Arendt, Paul Ricoeur, Eric Hobsbawm e incluso a Francis Fukuyama.

Tras una aclaratoria introducción sobre las pretensiones del estudio, se nos presenta el primer capítulo, que lleva por título Chronique d'une histoire annoncée, y está dedicado a la integración de la historia de las provincias romanas de la Península Ibérica en la Historia y la identidad nacional de España. Patrick le Roux analiza cómo la construcción del imaginario colectivo español se nutre de hitos fundacionales mirando hacia la antigüedad, en un caso paralelo al de Francia y su uso nacionalista de los conflictos entre Vercingetorix y César, de manera que para la historiografía tradicional, como la reflejada por Ramón Menéndez Pidal en su Historia de España, las diferentes vicisitudes de la conquista romana de la Península Ibérica constituirán, a falta de una figura unificadora como la del caudillo arverno, los primeros ejemplos de una identidad española en fase embrionaria. Siguiendo esta línea de pensamiento, los hispanos se caracterizarán por su carácter aguerrido y una pertinaz resistencia frente al invasor (quizá resulte aquí pertinente volver a citar a Vercingetorix o a la reina icena Boudica como muestra de un tópico identitario), demostrada a lo largo de un buen número de episodios durante los dos últimos siglos antes de la era cristiana, esto es, los protagonizados por Viriato, los numantinos, Sertorio, o los cántabros del noroeste. Estos rasgos todavía aparecerán más exacerbados cuando la abrumadora superioridad de fuerzas de Roma se acabe imponiendo, abocando a los resistentes a un irremisible destino trágico, cuya mejor representación es sin duda la antropofagia de los 
desesperados defensores de Numancia, aunque también resultan ilustrativos los casos de Viriato y Sertorio, que no pudiendo ser domeñados por las armas, lo fueron mediante la traición. Junto a este espíritu irredento, a través del cual también se justifica la ausencia de grandes jefaturas que pudieran dar lugar a grandes estados territoriales, el pueblo "español" por excelencia de la antigüedad, los iberos, aparecen retratados por la historiografía tradicional peninsular como un pueblo indígena culturalmente superior a cualquier otro de occidente excluyendo a las potencias colonizadoras. Sin embargo, completada la conquista, parece operarse un giro copernicano en la mentalidad de los indígenas, que adoptan las bondades de la latinidad y se suman entusiasmados a la tarea de la construcción del Imperio, aportando un "genio hispano" que los romanos aceptan gustosos, sabedores del valor de los habitantes de la Península. Así, algunos hispanos llegaron incluso a ser príncipes, como Trajano, quien llevó el Imperio a su máxima extensión, o Teodosio el Grande, el último gobernante de un Imperio Romano unificado. Ya en el siglo $\mathrm{V}$, el aislamiento de los territorios hispanos del resto de la romanidad prefigura la futura unidad política de España.

Frente a este discurso historicista, preponderante en nuestro país hasta los años setenta y hoy desechado por el mundo académico, que podríamos calificar como teleológico en el sentido de concebir la antigüedad hispana como un mero prólogo de una ulterior realidad política española, el autor procede a desmontar los tópicos antes referidos exponiendo las novedades de la investigación de las últimas décadas, como la total falta de carácter identitario de la provincia (papel que desempeña la comunidad urbana), o la ausencia de un lobby o "partido hispánico" en el Senado de Roma que aupara a Trajano al poder. El capítulo termina con una breve reflexión sobre cómo el estructuralismo y la escuela de los Annales han influido en el método de los historiadores de la segunda mitad del siglo XX.

La segunda parte del libro, titulada Le beurre et l'huile, ofrece una comparativa entre pueblos indígenas y romanos desde el punto de vista de estos últimos, tomando como hilo conductor el libro sobre la Península Ibérica (el tercero) de la Geografía de Estrabón. Aunque el propósito de esta obra es el conocimiento del territorio, se inscribe en la tradición griega de la geografía, por lo que recibimos tanta información de carácter etnográfico como geográfico (a costa de sacrificar exactitud en esta última), convirtiéndose así en un instrumento de especial valor para el historiador moderno. De hecho, Estrabón sigue un criterio de ordenación de los territorios de acuerdo a las divisiones étnicas (Turdetania, Celtiberia). No hay en toda su obra ninguna referencia a Iberia con una connotación unitaria más allá de lo exclusivamente geográfico, en contraste con los "esencialismos" hispánicos que pretendía ver la historiografía española de los siglos XIX y buena parte del XX.

La concepción griega del mundo, que divide las poblaciones entre bárbaros y civilizados, tiene un claro reflejo en la obra de Estrabón, que separa artificialmente la Península entre los territorios próximos al Mediterráneo, que han adoptado los usos y beneficios de la civilización traídos por fenicios, griegos y romanos, y el resto, especialmente el noroeste, que es representado como un epítome de la barbarie, y sus habitantes definidos por características opuestas a las de griegos y romanos, una identidad construida, evidentemente, mediante la alteridad. Así, el autor enumera los elementos que, a juicio de Estrabón, son definitorios de una y otra cultura. Mientras griegos y romanos adoran a divinidades conocidas, los indígenas del noroeste son ateos, o, utilizando el ejemplo que da nombre al capítulo y con el que el autor se detiene varias 
páginas, los pueblos civilizados utilizan el aceite para cocinar y los indígenas manteca. Quizá el principal rasgo definitorio de la civilización a ojos de un griego como Estrabón sea la habitación en comunidades de tipo cívico, único modo de vida en el que un hombre puede desarrollarse plenamente, lo que redunda en la oposición geográfica entre una Iberia urbana y una Iberia rural. Tan sólo la primera está integrada en la romanidad, aunque ambas forman parte de la economía-mundo ligada a la expansión de Roma. En definitiva, lo que Estrabón pretende demostrar en su obra es la superioridad de la civilización romana sobre las indígenas, y los beneficios que éstos han recibido de la conquista de Roma. Todo ello desde una perspectiva etnocéntrica y plagada de arquetipos simplificadores, todo lo contrario a lo que entendemos hoy en día por un estudio antropológico. Esta concepción de Hispania es fruto de una total asimetría de las fuentes históricas, producto a su vez de la falta de relatos desde el punto de vista de los indígenas conquistados, quienes carecen de una tradición historiográfica escrita propia.

El tercer capítulo de la obra se titula Pierres qui parlent et ne parlent pas, y está destinado por completo a la epigrafía y sus contribuciones al conocimiento de la antigüedad romana. Siendo el autor especialista en esta disciplina, este capítulo presenta un carácter marcadamente más técnico que el resto, aunque ello no supone ni mucho menos que el lector deba ser competente en la materia para comprender el contenido. Comienza advirtiendo del riesgo que supone tomar las fuentes epigráficas como meros instrumentos con los que confirmar lo expuesto por los autores clásicos, defendiendo la autonomía de la disciplina y la ausencia de "apriorismos literarios" en la mente del epigrafista. A diferencia de las fuentes literarias, con un acentuado sesgo favorable a las elites, la epigrafía nos permite asomarnos al mundo de los estratos humildes de la sociedad romana, otorgándonos muchos más protagonistas históricos y constituyendo la principal herramienta para elaborar prosopografías en la antigüedad. Asimismo, podemos acercarnos, debido a su carácter de fuente primaria, al conocimiento de las mentalidades y de la identidad con la que los individuos que encargaban que les fuera erigido un epígrafe querían autorrepresentarse. A continuación, se exponen con abundantes ejemplos algunos de los problemas de trabajar con inscripciones: raramente se hallan en el lugar donde se encontraban en la antigüedad, es habitual tener que lidiar con propietarios particulares que no siempre facilitan el estudio de las piezas, el valor intrínseco de algunos objetos inscritos, que los hace susceptibles de robo o tráfico en el mercado negro, o la excesiva compartimentación local y regional de los estudios de los materiales, que enmascara artificialmente con fronteras modernas continuidades que se producían en la antigüedad. Igualmente, hay que tener en cuenta, como en cualquier disciplina en la que se trabaje con un número reducido de ejemplares y se quieran extraer conclusiones de carácter general, que el gran número de piezas que no han llegado hasta nosotros podría contener información que invalide nuestra interpretación.

Otra cuestión tratada en esta parte es la elección de los materiales empleados en la fabricación de un epígrafe, que no es aleatoria, de forma que podemos esperar que materiales costosos, como el mármol, correspondan a piezas de singular importancia o a propietarios de extracción económica elevada. El mismo fenómeno se experimenta sobre otros materiales no pétreos, como el bronce, que se especializó en el grabado de documentos jurídicos. También hay espacio para hablar de los límites del conocimiento epigráfico, como la realidad concreta que se esconde detrás de determinados nombres, imposible de dilucidar sin una mayor información que la que presta la propia inscripción, caso de las divinidades indígenas de la Península Ibérica, ocultas tras oscuros teónimos locales. Tampoco es posible, como en ocasiones se ha pretendido, 
medir el índice de alfabetización de forma cuantitativa en el mundo romano a través del número de epígrafes de una determinada zona, ya que la gran mayoría de inscripciones fueron erigidas por miembros de los grupos sociales más acomodados, y además habría que obviar el propio carácter monumental de un epígrafe sobre piedra, destinado no sólo a ser leído, sino también a ser contemplado.

El cuarto capítulo del libro, Hadrien sans Italica, que versa obviamente sobre el emperador Adriano y su relación con su lugar de nacimiento, Italica, arranca con la interpretación tradicional que la historiografía española hacía sobre los gobiernos de los emperadores romanos de origen peninsular, esto es, Trajano, Adriano y Teodosio I, que eran presentados como una muestra de la importancia y la influencia de las provincias hispanas durante el Imperio. Tras esto, el discurso se centra ya exclusivamente en Adriano, nacido en la ciudad bética de Italica, cuna también de su inmediato predecesor Trajano, el primer emperador nacido fuera de Roma. El que un provincial pudiera alcanzar la cima del poder imperial da cuenta del inmenso avance que había experimentado la integración de las provincias en el sistema político-administrativo romano. Las provincias hispanas habían evolucionado sobremanera desde el panorama barbarie-civilización presentado por Estrabón en tiempos de Augusto, hasta el punto de que la propia Bética presentaba un paisaje tan urbanizado y romanizado como cualquier región de Italia. No fue pues casualidad que de este territorio provinieran los primeros príncipes extraitálicos. Sin embargo, la idea tan extendida hasta hace unos pocos años de la existencia de un clan o partido "español" que aupó a la púrpura a Trajano y a Adriano, debe ser desechada, ya que si fueron designados para tal honor fue por sus meteóricas carreras políticas y militares y por las alianzas que tejieron entre los senadores y otros altos funcionarios imperiales, independientemente de su origen geográfico. Incluso conocemos la existencia de senadores hispanos contrarios al nombramiento de Adriano.

La segunda parte del capítulo trata sobre la ciudad de Italica, fundada en 206 a. C. como colonia latina en el marco de la Segunda Guerra Púnica y transformada en municipio de derecho romano tras las guerras cesarianas. Era, como mucho, una ciudad de tamaño medio, muy inferior a Corduba o Tarraco, y habitada por unas dos mil personas. Sin duda la fuente principal de su fama en la antigüedad era haber sido la patria de Trajano y Adriano, siendo con este último con quien guardará mayor relación la ciudad a raíz de un cambio estatutario solicitado por la propia ciudad, como narra Aulo Gelio, mediante una embajada enviada a Adriano pidiendo cambiar su situación de municipio a colonia romana, estatuto más gravoso económicamente y anacrónico, pero también más prestigioso. El propio príncipe aconseja en contra de dicho cambio, pero finalmente lo concede, adoptando la ciudad en su honor el nombre de Colonia Aelia Augusta, reforzando así todavía más su lazo con el emperador. La motivación de la ciudad para querer adoptar un sistema fiscalmente desfavorable es considerarse una parte de Roma fuera de Italia (ficción ideológica de las colonias romanas), habiendo desarrollado una identidad colectiva fuertemente ligada a la romanidad (primer asentamiento romano en la Península Ibérica, patria del emperador), que les insta a diferenciarse del resto de sus vecinos provinciales.

El autor termina esta parte de la obra contraponiendo la figura del emperador Adriano que nos transmiten las fuentes clásicas, autoritario, vengativo y enfrentado permanentemente con el Senado de Roma, con el retrato que se tiene popularmente de él, forjado sobre todo a partir de las Memorias de Adriano de Marguerite Yourcenar, 
como un príncipe pacífico, viajero e intelectual. Esta semblanza es una construcción histórica moderna del personaje que en nada se corresponde con los datos que la investigación de la antigüedad permite vislumbrar.

Los dos últimos capítulos del libro, Dans l'atelier de Clio y Noveaux chemins?, son los que tienen un componente historiográfico más acentuado y una perspectiva más personal del propio autor, el primero centrado en la construcción del discurso histórico y el método de trabajo y el segundo en la búsqueda de nuevos enfoques de investigación en la antigüedad romana.

De acuerdo con Patrick Le Roux, la Historia Antigua se ha visto menos influenciada que otras materias históricas por las innovaciones procedentes de otras disciplinas, fundamentalmente la filosofía, la antropología y la sociología. Se han hecho menos análisis desde la Historia Total o estructuralistas de la antigüedad que de los periodos posteriores, y la Historia Antigua no parece particularmente preocupada por la Teoría de la Historia. Probablemente el método de trabajo ligado a la interpretación de las fuentes clásicas no fomente la adopción de innovaciones metodológicas, puesto que permite avanzar en el conocimiento histórico a través de los nuevos descubrimientos literarios o arqueológicos sin necesidad de un nuevo enfoque histórico de la disciplina.

Dentro del propio estudio de la historia romana, hay un desigual tratamiento de periodos y temas, habiéndose estudiado con mucha mayor profusión la República Tardía y el Alto Imperio, que los periodos anteriores y posteriores a ellos. El mismo análisis se puede hacer con la muy bien conocida historia política y administrativa frente a la menos trabajada historia económica, o, en el marco de la epigrafía, con las piezas de carácter jurídico, objeto de estudio tanto de historiadores de la antigüedad como de investigadores del Derecho Romano, y las inscripciones ligadas a actividades productivas, mucho menos trabajadas. En los aspectos de historia económica y social de Roma se produce actualmente un abuso del término "larga duración", que lleva a simplificaciones e impide la realización de estudios parciales en profundidad, que desmonten esa perspectiva monolítica. Un panorama similar podemos observar en los estudios de las provincias, donde sólo ahora parece que se empieza a incidir en las particularidades de los diferentes territorios y sus relaciones con Roma y entre ellas, o los diversos procesos de creación de estas circunscripciones. Fueron muy importantes los estudios que desde los años sesenta y haciéndose eco de su realidad, introdujeron en el estudio del mundo provincial romano los términos "colonialismo" y "resistencias culturales", en contraposición a la imagen del "milagro romano" que se tenía hasta entonces. Pero sin duda, el concepto que más ha cambiado y que todavía hoy se halla en constante reformulación, es el de romanización, que oscila entre aquellos que defienden un proceso uniforme para todo el Imperio y quienes por el contrario ven tantas romanizaciones como provincias.

Víctor Simón Castejón

vsimon@unizar.es

Universidad de Zaragoza (España) 
Fecha de recepción: 14 de diciembre de 2012

Fecha de aceptación: 18 de diciembre de 2012

Publicado: 31 de diciembre de 2012

Para citar: Víctor Simón Castejón, "Patrick Le Roux, Mémoires hispaniques. Essai sur la pratique de l'Histoire, Madrid: Casa de Velázquez, 2012, 212 pags.", Historiografías, 4 (julio-diciembre, 2012): pp. 116-121, http://www.unizar.es/historiografias/historiografias/numeros/4/castejon.pdf 